\title{
Hybrid Laser Technology for Creation of Doped Biomedical Layers
}

\author{
Miroslav Jelinek ${ }^{1,2 *}$, Lucie Bacakova ${ }^{3}$, Jan Remsa1,2, Tomas Kocourek1,2, \\ Jan Miksovsky',2, Petr Pisarik1,2, Marta Vandrovcova ${ }^{3}$, Elena Filova ${ }^{3}$, Sarka Kubinova ${ }^{4}$ \\ ${ }^{1}$ Institute of Physics ASCR, Prague, Czech Republic \\ ${ }^{2}$ Faculty of Biomedical Engineering, Czech Technical University in Prague, Kladno, Czech Republic \\ ${ }^{3}$ Department of Biomaterials and Tissue Engineering, Institute of Physiology ASCR, Prague, Czech Republic \\ ${ }^{4}$ Institute of Experimental Medicine ASCR, Prague, Czech Republic \\ Email: "jelinek@fzu.cz
}

Received 6 November 2015; accepted 8 January 2016; published 12 January 2016

\begin{abstract}
Hybrid laser technologies for deposition of thin films and basic schemes of combination of pulsed laser deposition (PLD) with magnetron sputtering and RF discharges or two lasers or three laser deposition systems are presented. Experiences with deposition of chromium doped diamond-like carbon (DLC) films for coating of prostheses are described. Layers of different chromium concentrations were prepared using hybrid systems (PLD + magnetron sputtering or by double PLD). Results of physical and mechanical characterization of film properties and biomedical tests of trivalent and toxic hexavalent chromium are given. Experiences with double laser deposition of DLC layers doped with silver are also mentioned.
\end{abstract}

\section{Keywords}

Hybrid Laser Technology, Biomaterials, Thin Films, Doped Layers, DLC

\section{Introduction}

For creation of thin films of biomaterials, technologies like PVD (Physical Vapor Deposition) and CVD (Chemical Vapor Deposition) are used. For fabrication of complicated nanocomposites the focus is on technologies like sputtering and laser deposition.

Lasers can be employed in a various techniques used for the fabrication of biomaterials, such as drilling, cutting, welding, annealing and hardening. For the coating of implants, development of biosensors, detectors, nanomaterials, and creation of new components, the laser techniques such as laser ablation, surface modification, laser induced forward transportation (LIFT) and Laser chemical vapor deposition (LCVD) are used. A frequently applied method in material research is the laser deposition method, usually called PLD. This method allows the fabrication of a wide scale of inorganic coatings and layers, including selected organic layers [1] [2]. For organic materials a modification of PLD called MAPLE (Matrix assisted pulsed laser evaporation) is frequently

\footnotetext{
${ }^{*}$ Corresponding author.
}

How to cite this paper: Jelinek, M., Bacakova, L., Remsa, J., Kocourek, T., Miksovsky, J., Pisarik, P., Vandrovcova, M., Filova, E. and Kubinova, S. (2016) Hybrid Laser Technology for Creation of Doped Biomedical Layers. Journal of Materials Science and Chemical Engineering, 4, 98-104. http://dx.doi.org/10.4236/msce.2016.41014 
applied. PLD is a very flexible method, with a wide range of possible deposition conditions, a wide choice of applicable materials and high deposition rates. The flexibility of PLD allows the deposition of multilayered structures in situ, in a single technological step.

In this contribution we introduce PLD and basic hybrid PLD technologies, including applications for creation of DLC films doped with chromium or silver.

\section{Laser Thin Film Hybrid Technologies}

In this chapter we describe principle of PLD for synthesis of inorganic and organic thin films and basic hybrid PLD arrangements. PLD equipment consists of a laser and a deposition chamber. The laser beam is focused onto a target, which is placed in a vacuum chamber. The chamber input window must have a large diameter to enable the laser beam to scan the target and to ensure standard conditions for material evaporation. The stream of material from target (plasma plume) is directional and perpendicular to the target. Material condenses on the substrate, which is placed on a heating element. Further advantages of PLD are that it allows stoichiometric deposition, it uses a relatively simple and economical pumping system $\left(10^{-3} \mathrm{~Pa}\right)$, and it enables a simple deposition of multilayers. The PLD deposition scheme and a photo of a real deposition chamber are shown in Figure 1.

MAPLE is a relatively new method of physical vapor deposition (PVD), similar to PLD. MAPLE is used for the fabrication of organic layers. One drawback of using standard PLD to produce films is that the direct ablation of target can be stressful to fragile materials, which may consequently break during the process. Maple was developed to decrease the photochemical damage caused by the direct interaction of the UV laser light with the organic or biomaterial target and to overcome the difficulties in solvent-based coating technologies, such as in homogeneous films, inaccurate placement of material, and difficult or incorrect thickness control. The process uses a low fluence pulsed UV laser and a cryogenically-frozen composite target consisting of a dilute mixture of the material to be deposited and a high vapor-pressure solvent—see Figure 2.
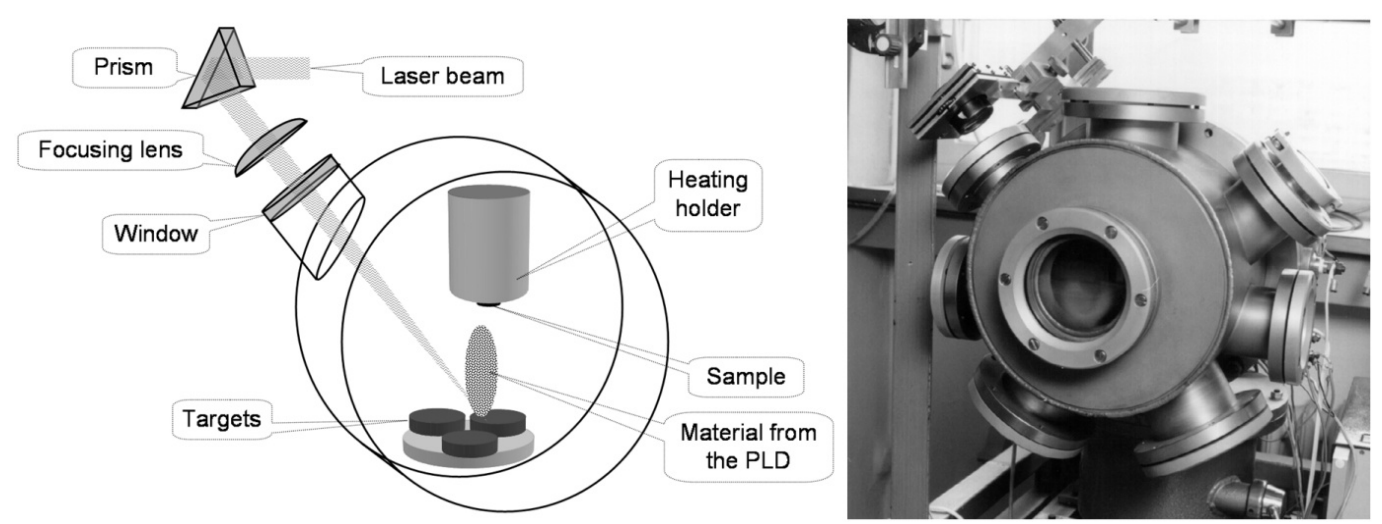

Figure 1. Scheme of PLD deposition chamber (left) and photo of real chamber (right).
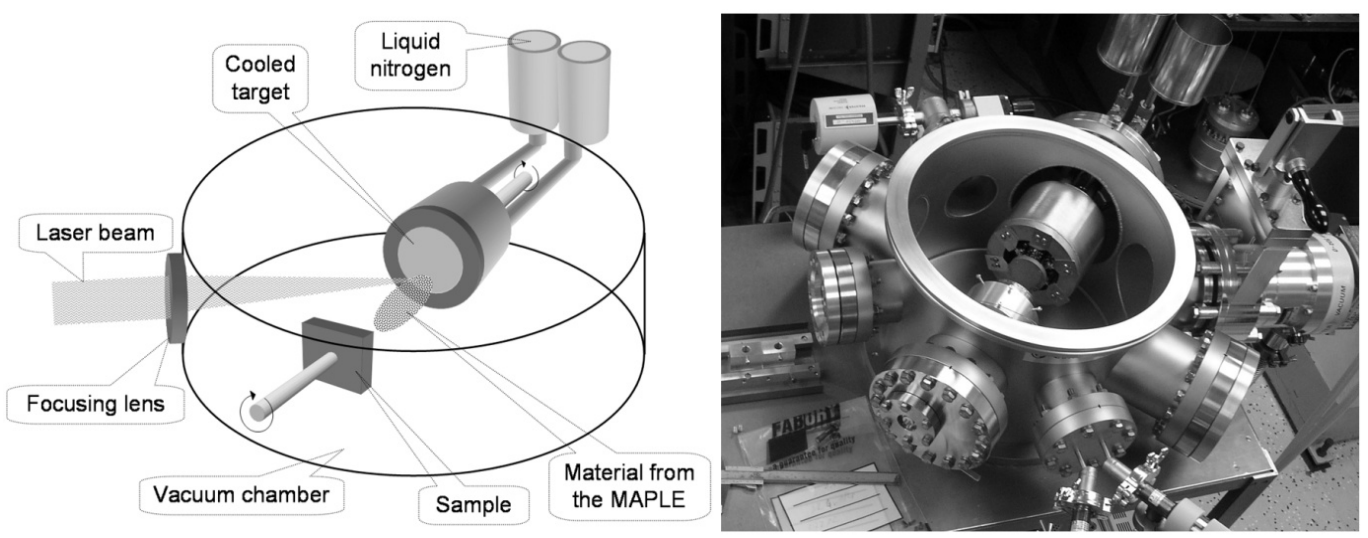

Figure 2. Scheme of cryogenic MAPLE deposition system (left) and photo of MAPLE chamber (right). 
To overcome some of the limitations of deposition techniques, to find new possible deposition methods, a several novel combined deposition techniques are currently under development. Hybrid technologies are used, for example, to improve surface structure, stoichiometry, morphology and adhesion, and to enable the fabrication of nanocomposites and doped materials. Examples of some hybrid laser based deposition schemes are in Figures 3-7.

The hybrid deposition techniques are steadily improved to meet the higher technological requirements. Example of hybrid PLD of a new generation is in Figure 8. Chamber enables simultaneously running PLD, double PLD and ion beam, and magnetron deposition.

\section{Biomaterial Research Using Hybrid Laser Technology}

Biomaterials, i.e. natural or artificial materials designed for application in biotechnologies and medicine, are widely used for replacing irreversibly damaged tissues in the human body.

Biomaterials have to meet the requirements for well-functioning and long-term durability of the implants, namely biocompatibility, good corrosion and fatigue resistance, wear resistance and biomechanical compatibility [3]. In this part we mention experiences and results with hybrid deposition of DLC films doped with chromium or silver.

a)

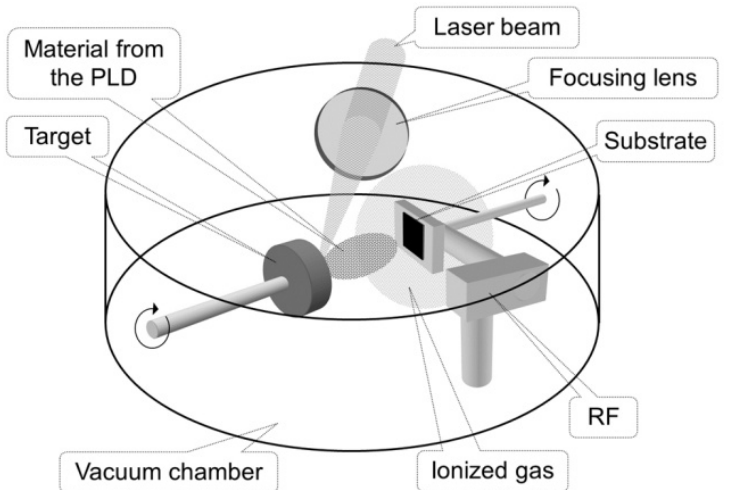

b)

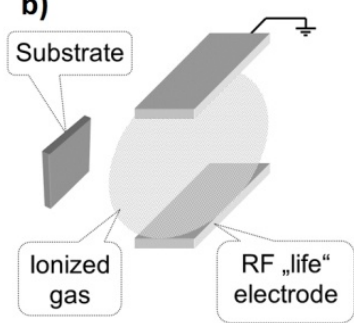

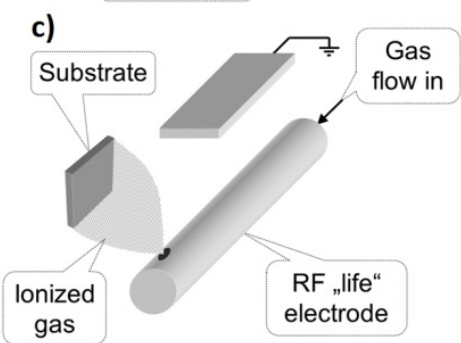

Figure 3. Scheme of hybrid deposition system chamber combining PLD with radiofrequency (a,b) and hollow cathode discharges (c).
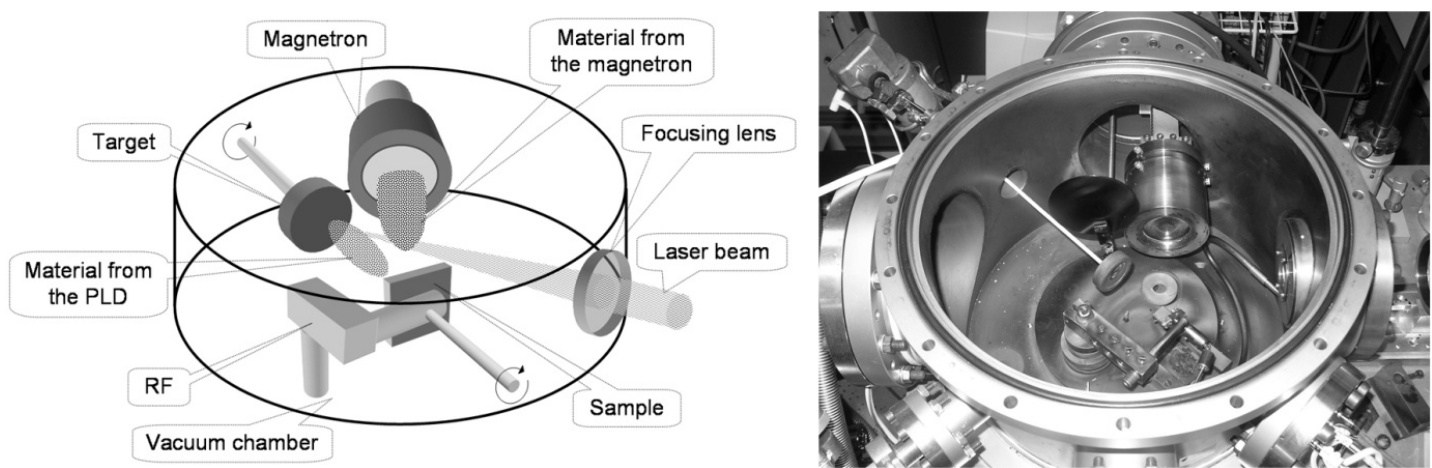

Figure 4. Scheme of hybrid PLD—magnetron deposition system (left) and photo of real chamber (right). 

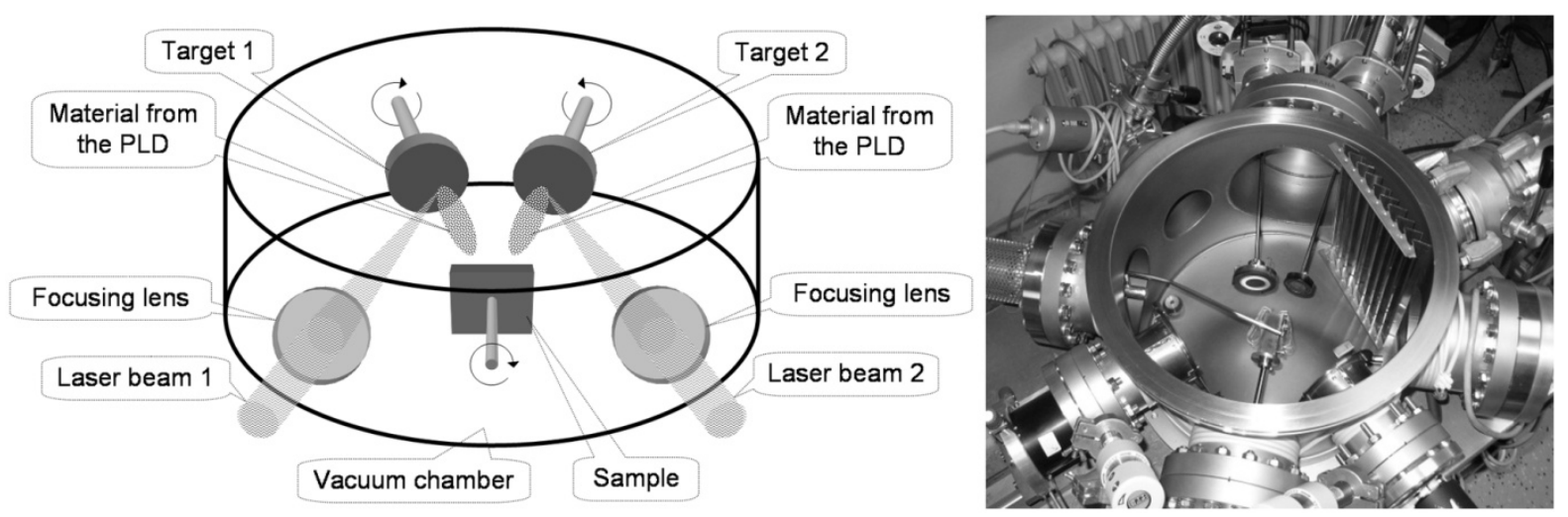

Figure 5. Scheme of hybrid PLD using two lasers and two targets.
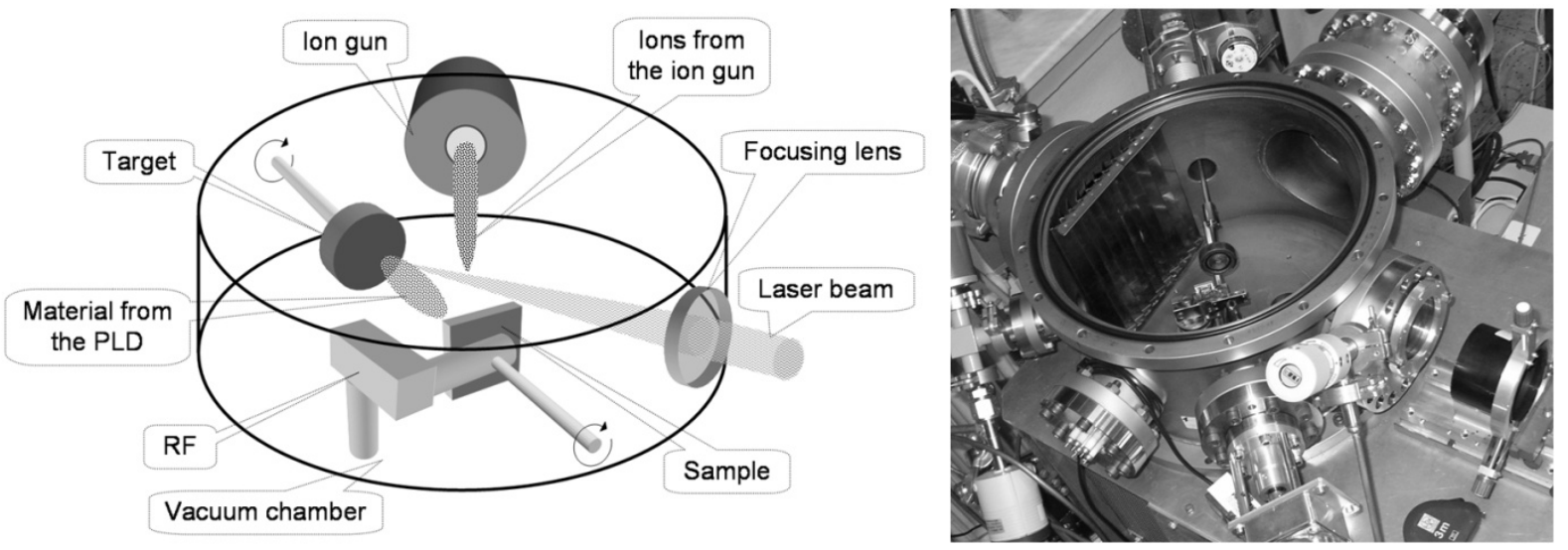

Figure 6. Scheme of hybrid PLD laser deposition combined with ion gun (left) and photo of system (right).

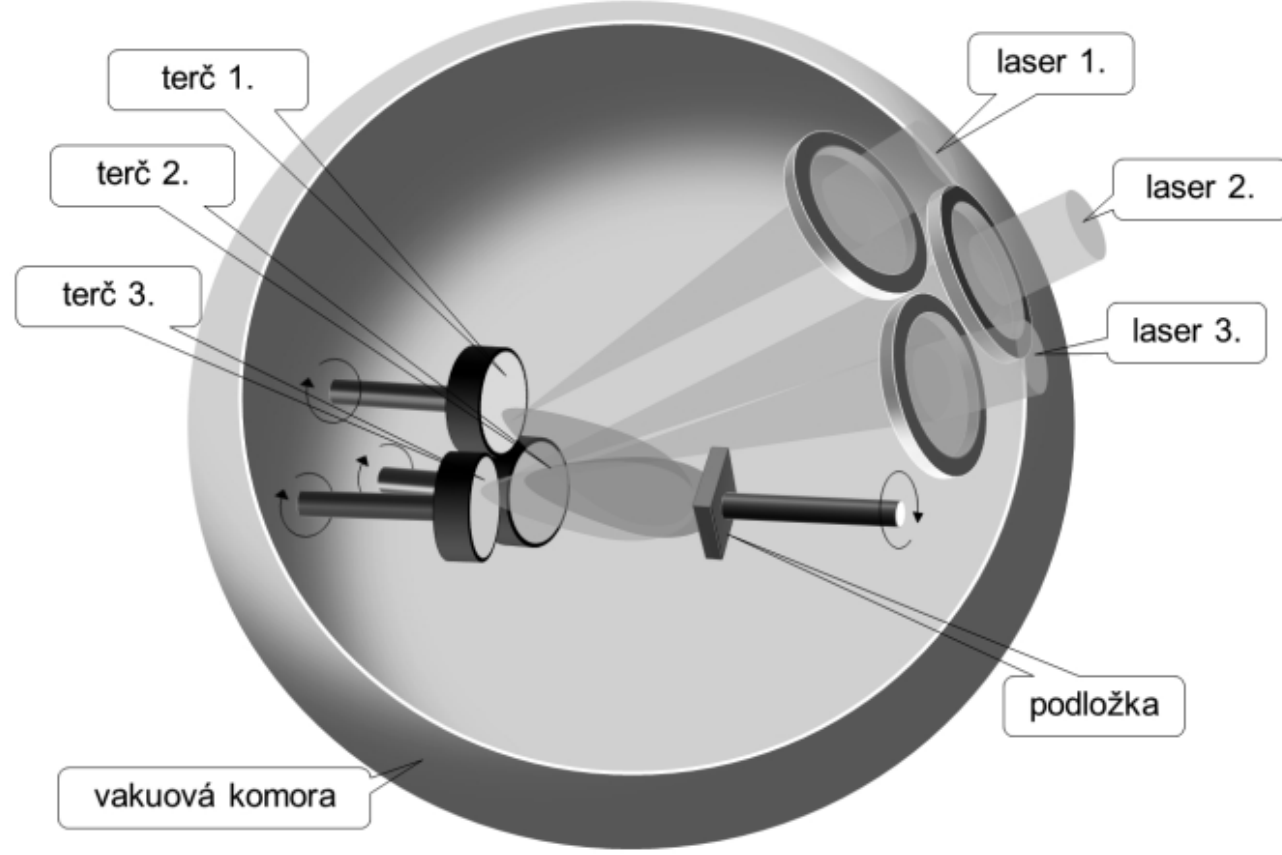

Figure 7. Hybrid laser based deposition method combining simultaneous deposition of doped and nanocomposited materials from three targer, using three lasers. 

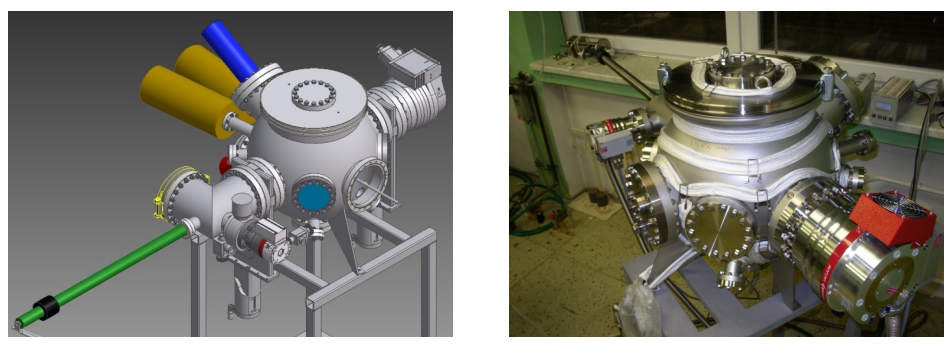

Figure 8. PLD chamber with load lock (base vacuum $5 \times 10^{-6} \mathrm{~Pa}$, diameter of $450 \mathrm{~mm}$, TBM 700 l/s, TBM 80 l/s—for load lock) (left—schema, right—photo of real chamber).

\subsection{Cr: DLC Layers}

Chromium is used as a dopant for improvement of adhesion of DLC films. Chromium is also a common part of stainless steels and alloys used for implants (up to $30 \mathrm{wt} \%$ ) [4]. Chromium-doped diamond-like carbon (DLC) layers were prepared by a hybrid technology using a combination of PLD and magnetron sputtering (2 inch. K. J. Lesker) or PLD and PLD (double PLD deposition—-two KrF excimer lasers were used).

\subsubsection{Cr: DLC Prepared by Double PLD Deposition}

One $\mathrm{KrF}$ excimer laser was used for ablation of carbon and the second for ablation of chromium. The flows of targets materials were regulated by lasers repetition rates. Chromium content was in region from zero to 18 at\%. Roughness was studied by mechanical profilometer or by AFM. Roughness of doped films was comparable with the roughness of undoped DLC films. With increase of chromium content the roughness was slightly increasing [5] or decreasing [6]. Roughness Ra increased from about $0.62 \mathrm{~nm}$ to $1 \mathrm{~nm}$ (or decreased from $10 \mathrm{~nm}$ to $5 \mathrm{~nm}$ ]. With increasing chromium content the hardness changed from $52 \mathrm{GPa}$ to $17 \mathrm{GPa}$ but adhesion critical force $\mathrm{L}_{\mathrm{c} 3}$ increased from $6 \mathrm{~N}$ to $19 \mathrm{~N}$ [5] [6]. The contact angle (CA) of undoped DLC was $70.6^{\circ}$. For chromium doped layers changed from $87^{\circ}$ to $93^{\circ}$. Surface free energy (SFE) gamma $T$ for undoped DLC layers was $43 \mathrm{mN} / \mathrm{m}$ and decreased for chromium doped layers to $31 \mathrm{mN} / \mathrm{m}$. No significant changes of SFE were observed for doped layers, the same is valid for zeta potential. Bacterial efficiency was low for all doped (undoped) layers [6].

\subsubsection{Cr: DLC Prepared by PLD + Magnetron}

Content of chromium was changed by magnetron power and of carbon by laser repetition rate. Measured chromium content in DLC layers was different for XPS measurement (in region from zero to 7 at\%) and Wavelength dependence X-ray analysis measurement (zero to 16 at\%). Films were very smooth. No reasonable differences in morphology were found between undoped and doped layers. Roughness Ra was in region from $0.2 \mathrm{~nm}$ to 0.7 $\mathrm{nm}$ (highest chromium concentration). Contact angle for water changed from $79^{\circ}$ (DLC) to $102^{\circ}$ (7 at\% of $\mathrm{Cr}$ ). The SFE decreased with chromium doping from $34 \mathrm{~J} \cdot \mathrm{m}^{-2}$ (DLC) to $28 \mathrm{~J} \cdot \mathrm{m}^{-2}$ (7 at\% Cr). The films do not exhibit any antimicrobial effect against both tested (positive and negative) bacterial strains [7]. From X-ray photoelectron spectroscopy follows that the chromium was present predominantly in its trivalent form. This form has been considered as relatively non toxic. Small amount of toxic, hexavalent, chromium was also found. The population density of Saos-2 cells was the highest in samples containing higher concentrations of chromium 7.7 and 10 at\%. However, this seemingly positive cell behavior could be associated with the risk of dedifferentiation and oncogenic transformation of cells [8] [9].

\subsection{Ag: DLC Layers Prepared by Double PLD}

Silver-doped DLC has been reported that these coating is able to reduce the residual stress state (thus improving the coating's adhesion to different substrates), to provide antibacterial properties and to improve the tribological behavior. AgDLC coatings have been pointed as an effective coating for biomedical implants due to their good corrosion resistance and wear resistance, antibacterial activity and hemocompatibility. The amorphous carbon coatings containing about 5.5 at\% of Ag were not toxic against mouse MC3T3 osteoblastic cells [10] [11].

Our Ag: DLC layers were prepared by double PLD deposition using two KrF excimer lasers. Concentration of silver dopant (from zero to 10 at\%) was easily regulated by changing of repetition rates of lasers, which ablated silver or carbon from targets. Results of antibacterial testing using Gram-negative Pseudomonas aeruginosa and Gram-positive Staphylococcus aureus bacteria are in Figure 9. 


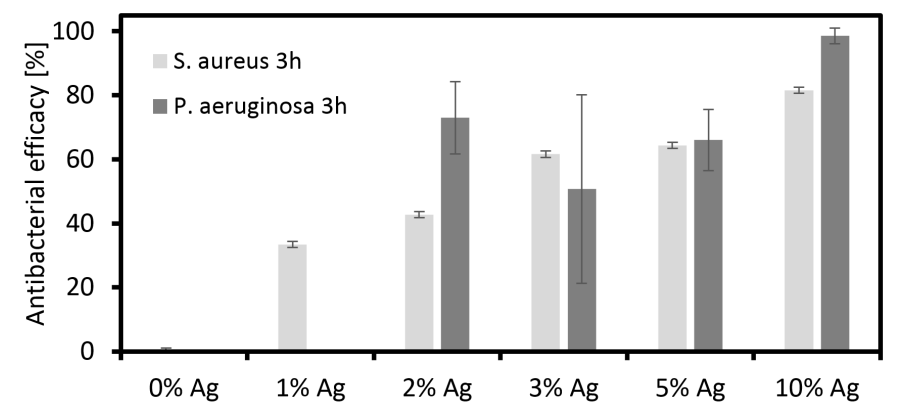

Figure 9. Bacterial inhibition on Ag: DLC layers after 3 h treatment in positive and negative bacterias [12].

\section{Conclusions}

We present laser technologies for preparation of thin films, with focus on biocompatible materials and hybrid deposition techniques. Schemes for deposition of inorganic films as PLD, and organic films as MAPLE, are mentioned. Hybrid laser based technologies for production of nanocrystalline, doped, nanocomposite and gradient layers are presented. Various hybrid arrangements as PLD + magnetron deposition, RF discharges, and double or triple PLD are presented.

Examples of applications of hybrid systems for study of doped biocompatible DLC coating using PLD + magnetrom and double PLD are described. Results of deposition of chromium doped DLC and silver doped DLC are discussed. Using various technologies also various films parameters can be preferably reached. Layers of Cr doped DLC prepared by magnetron were smoother, contact angle was higher and surface free energy was lower in layers prepared by PLD + magnetron, compared to double PLD. The preparation of layers of small dopant concentrations maybe sometimes better to arrange with double PLD because of PLD + magnetron exhibit instabilities in glow magnetron discharge for low magnetron power (needed for deposition of low dopant concentrations).

\section{Acknowledgements}

The work was supported by the Grant Agency of the Czech Technical University in Prague, grant No. SGS14/168/OHK4/2T/17 and by Grant Agency of the Czech Republic (grant GA-15-05864S and GA-1501558S).

\section{References}

[1] Chrisey, D.B. and Hubbler, G.K. (1994) Pulsed Laser Deposition of Thin Films. John Wiley and Sons Inc., New York.

[2] Eason, R. (2006) Pulsed Laser Deposition of Thin Films, Applications-Led Growth of Functional Material. John Wiley and Sons Inc, New York. http://dx.doi.org/10.1002/0470052120

[3] Geetha, M., Singh, A.K., Asokamani, R. and Gogia, A.K. (2009) Ti Based Biomaterials, the Ultimate Choice for Orthopaedic Implants-A Review. Progress in Materials Science, 54, 397-425. http://dx.doi.org/10.1016/j.pmatsci.2008.06.004

[4] Ratner, B.D., Hoffman, A.S., Schoen, F.J. and Lemons, J.E. (2013) Biomaterials Science: An Introduction to Materials in Medicine. 3rd Edition, Academic Press. http://dx.doi.org/10.1016/B978-0-08-087780-8.00148-0

[5] Písařík, P., Jelínek, M., Kocourek, T., Zezulová, M., Remsa, J. and Jurek, K. (2014) Chromium-Doped Diamond-Like Carbon Films Deposited by Dual-Pulsed Laser Deposition. Applied Physics A, 117, 83-88. http://dx.doi.org/10.1007/s00339-013-8206-1

[6] Jelínek, M., Písařík, P., Kocourek, T., Zemek, J., Kotzianova, A., Jurek, K., Miksovsky, J. and Luxbacher, T. (2014) Preliminary Comparative Study of Laser-Prepared DLC and Cr-Doped DLC for Bacteria Adhesion. Applied Physics A, 116, 1437-1443. http://dx.doi.org/10.1007/s00339-014-8256-Z

[7] Jelínek, M., Kocourek, T., Zemek, J., Mikšovský, J., Kubinová, Š., Remsa, J., Kopeček, J. and Jurek, K. (2015) Chromium-Doped DLC for Implants Prepared by Laser-Magnetron Deposition. Materials Science and Engineering: C, 46, 381-386. http://dx.doi.org/10.1016/j.msec.2014.10.035 
[8] Jelinek, M., Zemek, J., Vandrovcova, M., Bacakova, L., Kocourek, T., Remsa, J. and Pisarik, P. (2016) Bonding and Bio-Properties of Hybrid/Magnetron Cr-Enriched DLC Layers. Materials Science and Engineering: C, 58, 1217-1224. http://dx.doi.org/10.1016/j.msec.2015.09.006

[9] Filova, E., Vandrovcova, M., Jelinek, M., Zemek, J., Houdkova, J., Remsa, J., Stankova, L. and Bacakova, L. (2015) Differentiation of Saos-2 Osteoblast-Like Cells on Chromium-Doped Diamond-Like Carbon Coatings. PLOS ONE, submitted.

[10] Manninen, N.K., Galindo, R.E., Carvalho, S. and Cavaleiro, A. (2015) Silver Surface Segregation in Ag-DLC Nanocomposite Coatings. Surface and Coatings Technology, 267, 90-97. http://dx.doi.org/10.1016/j.surfcoat.2014.12.029

[11] Endrino, J.L., Escobar Galindo, R., Zhang, H.S., Allen, M., Gago, R., Espinosa, A. and Anders, A. (2008) Structure and Properties of Silver-Containing a-C(H) Films Deposited by Plasma Immersion Ion Implantation. Surface and Coatings Technology, 202, 3675-3682. http://dx.doi.org/10.1016/j.surfcoat.2008.01.011

[12] Písařík, P., Jelínek, M., Remsa, J., Mikšovský, J., Zemek, J., Jurek, K., Lukeš, J., Šepitka, J. and Kubinová, Š. (2016) Antibacterial, Mechanical and Surface Properties of Silver Doped Diamond Like Carbon Prepared by Dual Pulsed Laser Deposition. Diamond and Related Materials, submitted. 